\title{
Open Access \\ Wichtige Publikationsform in der Medizin
}

Durch die Publikationsform „Open Access“ soll wissenschaftliche Fachliteratur kostenfrei und allen Interessierten öffentlich frei zugänglich sein. Lediglich hinsichtlich Wiederabdruck, Verteilung oder Urheberrecht soll den Autoren die Kontrolle über ihre Arbeit belassen und ihr Recht gesichert werden, dass ihre Arbeit angemessen anerkannt und zitiert wird, formulierte 2002 beispielsweise die Budapester Open Access Initiative (www.soros.org/openaccess).

Die „Open-Access“-Idee hat sich auf der Basis verschiedenster Dynamiken entwickelt. Beispielsweise entstand 2001 bei Chefredakteuren und Herausgebern etlicher hochrangiger internationaler Medizin-Journale die Überzeugung, dass das klassische Peer-Review-System nicht mehr geeignet ist, Publikationen vor dem unberechtigten Zugriff von Lobby-Interessen vor allem aus der Pharmaindustrie zu wahren und sich damit überholt hat (siehe Notfall \& Hausarztmedizin 2006; 32 (4): 152-153). In der Physik wiederum wurde schon in den 90er Jahren gefordert, mit freier Online-Publikation den wissenschaftlichen Austausch zu beschleunigen.

\section{Regelung in den USA}

Die US-Regelung sieht vor, dass alle Publikationen, die von den US National Institutes of Health (NIH, www.nih.gov) finanziert werden, spätestens 12 Monate nach ihrer Veröffentlichung von der Öffentlichkeit frei und ohne Beschränkungen im Internet verfügbar sein müssen (publicaccess.nih.gov). Einen bedeutenden Einfluss auf die Formulierung der Open Access-Grundsätze haben schließlich auch Bürgerrechtsgruppen wie z. B. die Electronic Frontier Foundation (EFF, www.eff.org). Nach deren Auffassung seien wissenschaftliche Publikationen das Ergebnis einer oft von allen Bürgern finanzierten Arbeit, deren Verwertung nicht alleine privaten Verlagen zustehe. Abgesehen von der Vorstellung, dass wissenschaftliche Forschungsergebnisse im Interesse des Fortschritts und des gesamtgesellschaftlichen Nutzens keiner Zugangsbeschränkung unterliegen sollten. Trotzdem wurde im Februar 2009 ein Gesetzesvorschlag im US-Repräsentantenhaus eingebracht, der das kostenfreie Publizieren staatlich finanzierter Forschungsergebnisse rückgängig machen soll. Alle oben genannten Kreise, einschließlich von 33 US-Nobelpreisträgern, haben sich innerhalb kurzer Zeit gegen diesen Vorstoß ausgesprochen. Die Entscheidung steht noch aus.

\section{Regelung innerhalb Europa}

Innerhalb der Medizin ist Open Access durch die Aktivitäten wichtiger OpenAccess-Zeitschriftenverlage und durch mehrere in der Forschung unverzichtbare Faktendatenbanken inzwischen relativ fest verankert (weitere Infos: www. open-access.net). Die oben genannten Regelungen zur Förderung von Open Access in den USA haben jedoch in anderen westlichen Ländern bislang kaum Widerhall gefunden, zumindest nicht was die gesetzliche Übertragung entsprechender US-Regelungen auf die Wissenschaftsförderpolitik angeht. Immerhin unterstreicht der EU-Ministerrat, „die Wichtigkeit, den Lesern wissenschaftliche Ergebnisse aus öffentlich finanzierter Forschung kostenlos und unter wirtschaftlich tragbaren Bedingungen im Internet zur Verfügung zu stellen, u. a. durch freie Zugänglichkeit nach einem festgesetzten Verwertungszeitraum“ (Pressemitteilung, 22./23.11.2007).

\section{Open-Access-Verlage}

Public Library of Science

Die Public Library of Science (PLoS, www.plos.org) ist ein nichtkommerzieller Open Access-Verlag, der 2001 unter maßgeblicher Beteiligung des MedizinNobelpreisträgers und ehemaligen NIHDirektors Harold Varmus gegründet wurde, um qualitativ hochwertige Open Access-Zeitschriften herauszubringen. Als erste Zeitschrift wurde im Oktober 2003 die Zeitschrift PLoS Biology gegründet, gefolgt von PLoS Medicine in 2004, beide mit Impact-Faktor. Ihnen folgten etliche weitere Zeitschriften. Die PLoS-Zeitschriften finanzieren sich zum Teil über Autorengebühren.
BioMed Central

BioMed Central (www.biomedcentral. com) ist ein 1998 gegründeter, unabhängiger britischer Open Access-Verlag, der etwa 190 Open Access-Zeitschriften publiziert. Zahlreiche Zeitschriften von BioMed Central haben einen offiziellen Impact-Faktor. Die Zeitschriften von BioMed Central finanzieren sich ebenfalls über Autorengebühren oder alternativ über Mitgliedschaften.

German Medical Science

German Medical Science (www.egms. de) ist ein Portal für die Open AccessPublikation von Zeitschriften, Kongressen und Forschungsberichten. Es wurde 2003 als Gemeinschaftsprojekt der Arbeitsgemeinschaften der Wissenschaftlichen Medizinischen Fachgesellschaften (www.uni-duesseldorf.de/awmf), der Deutschen Zentralbibliothek für Medizin (ZB MED, www.zbmed.de) und des Deutschen Instituts für Medizinische Dokumentation und Information (DIMDI, www.dimdi.de) gegründet. Zurzeit erscheinen fortlaufend 13 Zeitschriften (Stand März 2009), zu denen auch die gleichnamige, das Gesamtgebiet der Medizin abdeckende Zeitschrift „GMS German Medical Science - an Interdisciplinary Journal“ gehört. Herausgeber und verantwortlich für das Peer Review der Zeitschriften sind jeweils die beteiligten medizinischen Fachgesellschaften. Die Finanzierung erfolgt über Mitgliedsbeiträge der beteiligten Fachgesellschaften, sowie über Spenden.

\section{Zeitschriften-Listen}

Das Directory of Open Access Journals (www.doaj.org) weist für die Bereiche Zahnmedizin 40, Medizin 279, Pflegewissenschaft 27 und Public Health 121 Zeitschriften aus. Zusätzliche Informationen für den Bereich der Medizin liefert die Internetseite Free Medical Journals (www.freemedicaljournals.com). Die Sammlung ist jedoch teilweise unbefriedigend gepflegt.

\section{Korrespondenz}

Rainer H. Bubenzer (DJV, KdM)

Medizin- und Wissenschaftsjournalist

Multi MED vision - Berliner Medizinredaktion Lützowstraße 47

10785 Berlin

Fax: 030/806136-80

Rainer@Bubenzer.com 